\title{
Time Pressure and the Extent of Homophily in the Workplace Communications of Architecture and Engineering Design Teams
}

\author{
Lindsey Walker ${ }^{1}$ and Bryan Franz ${ }^{2 *}$
}

\begin{abstract}
Workplace collaboration depends on communication and is critical in building design. For large, highly diverse teams, such as those found in architecture and engineering (A/E) who work under timebased deadlines, homophily — or the tendency to seek interactions with others of similar backgrounds and values - can play a role in how individuals communicate. Homophily is potentially damaging to teams that must coordinate information from a diverse membership, since communication may become less likely to occur across disciplines. Therefore, this research examines the extent to which a sampled $\mathrm{A} / \mathrm{E}$ team exhibits homophily in their information exchanges across multiple communication media, when under the moderating effect of two different levels of time pressure. The study uses a social network analysis of the communication patterns in an 18-member studio team working for a national A/E firm located in the southeastern United States. The results show some evidence of homophily as a predictor of information exchanges when controlling for the hierarchical ties within the studio team and the physical distance between its members in the office. In a low time pressure work environment, face-to-face communication was more likely when members were of the same gender. This effect was not present when the team was under high time pressure, where face-to-face interactions were instead more likely between members of the same discipline. Homophily in phone communication was found in the generational similarity of team members, regardless of time pressure. There was little evidence that homophily was a predictor of email communication. These results have implications for the design of studio workplaces that support information-rich interactions, the assignment of individual designers to project teams that are more likely to interact with co-workers from different backgrounds, and organizational policy regarding the use of specific communication media based on the project schedule and time pressure.
\end{abstract}

\section{Keywords}

Social network analysis, SNA, homophily, team communication, time pressure

\footnotetext{
${ }^{1}$ Department of Interior Design, University of Florida, Inner Road, Gainesville, United States

${ }^{2}$ M.E. Rinker, Sr. School of Construction Management, University of Florida, Gainesville, United States, bfranz@, ufl.edu (Corresponding Author)
} 


\section{INTRODUCTION}

The culture within many U.S. firms is becoming less hierarchical and, as a result, workplaces are now more open, with fewer enclosed offices (Becker, 2004). These spaces are designed to support multidisciplinary collaboration and are becoming increasingly important in knowledgebased work, such as building design. However, the effectiveness of that collaboration is often dependent on the richness of interactions between individuals in the workplace. These interactions can occur over a wide variety of media, including face-to-face conversations, video conferencing, phone calls, emails, and instant or text messaging. How individuals decide which medium to use has been the subject of extensive research. For example, media richness theory states that personalised media are generally more effective at conveying meaning because they are "rich" with cues (eg, tone of voice and facial expression) that provide information beyond the literal meaning of the message (Dennis and Kinney, 1998). When communicating, the theory predicts that individuals will choose a medium with an appropriate richness for the information being shared. Presumably, this choice will enable the most efficient form of information exchange.

For large and highly diverse architecture and engineering (A/E) teams, homophily can also play a role in whom individuals choose to communicate with (Mollica et al., 2003; Yuan and Gay, 2006). Homophily is the tendency for individuals to seek interactions with others of similar background, status, or values (McPherson et al., 2001). The proverb "birds of a feather flock together" is often used to describe these types of relationships. While homophily can facilitate cohesion within a team (Kim and Aldrich, 2006), the lack of exposure to contrary or dissimilar viewpoints can also limit the formation of new knowledge (Mollica et al., 2003; Yuan and Gay, 2006). For A/E teams, homophily is particularly damaging because it may restrict the flow of information across disciplines, leading to missed deadlines and rushed design decisions. Much of A/E teams' work consists of tasks that are delivered with some degree of urgency, with most team members feeling that they do not have enough time to complete those tasks. With the prevalence of schedule constraints, there is still little understanding about how communication in these teams responds to time pressure and even less about how time pressure may affect the extent of homophily driving those exchanges (Isenberg, 1981; Kelly and McGrath, 1985). Therefore, this research seeks to answer the question: To what extent does schedule pressure moderate the influence of homophily in workplace communications for an A/E team? Answering this question will explore whether the tendency of individuals to form homophilous relationships overrides their need for heterophily to address design problems on $\mathrm{A} / \mathrm{E}$ teams, under different time pressure work environments. Heterophily is the tendency for people to seek out or be attracted to those who are different from themselves.

This study conducted a social network analysis of an 18-member $\mathrm{A} / \mathrm{E}$ design studio team to examine the tendency of individual designers to communicate with co-workers of similar backgrounds during different time pressure intensities. Using an online survey, data was collected on the frequency of communication among the studio team members for three distinct media: face-to-face conversations, phone calls, and emails during two different weeks, and prior to the global COVID-19 pandemic. This data was used to model communication networks for each medium. Demographic data for each dyad of team members was then regressed on the communication networks to determine whether similarities in pairs of individuals predicted the network's topology when team experienced periods of low and high time pressure.

\section{BACKGROUND}

Many previous studies show the importance of homophily, as well as spatial proximity, and organisational structure, in workplace communication. Together, these strands of research recognise that the patterns of interaction among the team members result both from personal choice and the constraints established by the organisation. To holistically examine A/E team communication, additional strands of research must be combined. Although research on homophily and workplace communication has been carried out separately 
during the last few decades, no studies have analysed the combined effects of these sets of variables under the additional constraint of time pressure. The lack of prior research on the three-way interaction among homophily, workplace communication, and time pressure introduces the opportunity to examine $\mathrm{A} / \mathrm{E}$ teams who consistently interact with multiple communication media to deliver work under restrictive timelines (Brown and Miller, 2000).

\section{Homophily}

Within A/E teams, designers and engineers may be tempted to only collaborate with like individuals, specifically those of similar age, background, or values. This preference for people to interact or bond with others that are like themselves in socially significant ways is called homophily (McPherson et al., 2001). Often described as "birds of feather flock to together," the theory of homophily evolved from the similarity-attraction hypothesis (Byrne, 1971) and the theory of self-categorization (Turner, 1987), both of which predict that individuals tend to sort themselves based on their social or physical characteristics. Homophily is well-documented in social networking studies, confirming that similarity is a significant predictor of connections between individuals in workplace settings (Feld, 1982; Fischer, 1977; Fischer, 1982; McPherson et al., 2001; Sampson, 1984; Shrum et al., 1988; Yuan and Gay, 2006). Generally, there are two types of homophily: structural and choice. Structural homophily is the product of social constraints, such as a workplace that is predominantly a single gender. In contrast, choice homophily is the product of individual preferences for a certain trait or characteristic (Brashears, 2008).

Homophily has both positive and negative consequences for organisations. On the positive side, it can provide greater predictability in behaviour, a useful attribute for team building and member understanding (Lincoln and Miller, 1979). Homophilous bonds can also lead to relationships that are comforting and improve overall connection, raising perceived interpersonal trust between team members (Kalleberg et al., 1996; Kim and Aldrich, 2006). These bonds can provide a sense of solidarity during challenging times and facilitate increased easier team communication (Kalleberg et al., 1996;
Lincoln and Miller, 1979). Specific to A/E studio teams, these positive consequences of homophily can increase performance since frequent internal and external collaboration, elevated reasoning skills, and time-sensitive decision making are performed collectively as a team.

On the other hand, homophily can severely restrict the creation of new ideas within teams. Most researchers hand agree that the effects of homophily can be detrimental to teams and overall organisations, which require a variety of knowledge (Yuan and Gay, 2006). To create new knowledge, especially in creative and innovative design disciplines, individuals must interact with those who are dissimilar (Argote and Ophir, 2002; Argote, 1999; Lewis, 2000; Mollica et al., 2003). When communication only occurs among individuals with similar backgrounds or experiences, the likelihood of "groupthink" increases. Groupthink occurs when conformity or stability are elevated above innovation and involves collective decision-making that discourages creativity or individual accountability. The price of this stability is often diversity. However, diversity of thought is critical for design projects. Diversity can allow for exploration of a solution instead of convergence to an answer, and teams that display highly diverse attributes are shown to be more creative than homogeneous teams (Paulus and Nijstad, 2003). Thus, despite the benefits of homophily in creating cohesive teams, $\mathrm{A} / \mathrm{E}$ studio teams have a need for diverse ideas during their design process and cannot overly rely on individual similarity in their communication.

There are several drivers of homophily in teams. First, individuals find it easier to reach out to team members that are similar in age. Those of a similar age who typically fall within the same generational cohort can develop "a distinctive sub-cultural identity by virtue of having experienced the same historical events in the same ways at approximately the same time in their lives" (Alwin and McCammon, 2007, p. 231). A generation also shares momentous life events. These events include major wars, inventions, innovations, political and cultural movements, which shape generational characteristics that reflect the happenings in the world (Meier and Crocker, 2010). Additionally, Marsden (1987) identified that being of similar age increased the likelihood of individuals to 
performance and lead to social withdrawal (Charles and Veitch, 2002; Fried et al., 2001). In addition, spontaneous communication arising from proximity is not always desired, and can create unwelcome burdens on individuals focused on their work (Kraut et al., 2002).

\section{Organizational Structure}

The organisational structure of a company or firm establishes a set of possible encounters in the workplace, such as between a subordinate and their supervisor, peer to peer, and members of the same project team. Thus, much of the sorting and selfselecting of who individuals communicate with occurs through this formal structure. Specifically, the reporting structure within an organisation has been found to significantly affect the frequency of communication between pairs of individuals (Kleinbaum et al., 2008). In addition, an individual's position in the organisation effects the desirability of interactions. That is, individuals often seek to interact with those in leadership roles or of a higher status to obtain greater access to resources (Ibarra, 1992; Lin, 1982). When workplaces are designed based on the departmental role instead of project teams, individuals may be surrounded by like positions. While departmental grouping can support mentorship and develop applied skills, this form of workplace layout may not support cross-disciplinary efforts (Brass et al., 2004).

\section{Time Pressure and Communication}

The existence of deadline-based deliverables has a measurable effect on the performance of individuals and teams (Brown and Miller, 2000). Time as a constraint has been shown to have negative effects on decision-making and organisational outcomes (Argote et al., 1989; Brown and Miller, 2000; Chu and Spires, 2001). The time pressure created by these schedule deadlines can influence how teams communicate. Under high time pressure, teams have been found to work faster, but information is often exchanged sub-optimally and the quality of the teamwork can decrease (van der Kleij et al., 2009). This effect can vary by communication medium. Specifically, face-to-face communication has been associated with poorer performance in teams under 
high time pressure (Caballer et al., 2005), potentially undermining the benefits of reduced physical distance between team members. Chong et al. (2012) reached a similar conclusion that teams under high time pressure do not benefit from being in close proximity, and that time pressure can moderate the relationship between proximity and communication effectiveness.

As time pressure increases, there is an observable decrease in the number of team members communicating (Caballer et al., 2005). Decisions made during periods of high time pressure often reside with a few team members (Brown and Miller, 2000), with only the most communicative members increasing their frequency of information exchanges (Isenberg, 1981). The communication network of teams under deadline pressure becomes more centralised, with unequal participation by team members (Kelly and McGrath, 1985). This increase in centralization can result in closed mindedness to multiple alternatives, poor information processing, and group think (Caballer et al., 2005; Pelled et al., 1999, Isenberg, 1981). With the occurrence of groupthink, team members suppress their personal reservations and withhold relevant information to avoid conflict, maintain cohesiveness, and achieve an apparent group consensus (Janis, 1972, Isenberg, 1981).

\section{RESEARCH METHODS}

To examine the extent to which $\mathrm{A} / \mathrm{E}$ design teams exhibit homophily in their workplace, and to determine whether time pressure is a moderating factor, we analysed the communication networks of a single studio team. This studio team completed a survey questionnaire in which they answered demographic questions and quantified their communication patterns with each team member. The survey was distributed at two different time periods to capture data under both low and high time pressure work environments. From their responses, a quadratic assignment procedure (QAP) regression was performed for multiple communication media to determine whether similarities in team member demographics were predictive of their network topology.

\section{Sample Selection}

The selected studio team, as the unit of analysis in this research, worked for a national architecture, design, and engineering firm, with a regional office in the southeastern United States. Their office was established 20 years ago and employed 27 designers and support staff at the time of data collection. The studio team had multidisciplinary membership, including architects, interior designers, and project managers, who had experience primarily with designing corporate campuses, office buildings, lobby, and tenant suite buildouts. The size of the studio team was 18 people over the duration of the study. A layout of the office workplace for the studio team is provided in Figure 1. Members of the studio team worked independently and collaborated on projects of varying size and scope. Smaller projects typically required only one project manager and interior designer, while larger projects were given a larger staff, including a project manager, design principal, architect, and interior designer. Two ongoing large projects were underway at the time of data collection for both time periods. The studio principal determined team member assignments to projects based upon their experience, available time, and hourly cost. Additionally, studio team members were assigned to their workstations based on availability, the potential for project collaboration, and their expertise.

\section{Data Collection Instrument}

At the onset of the study, the authors first obtained the names of the studio team members, their email addresses, and a floor plan of the workplace in which they worked. A survey questionnaire was then developed in Qualtrics to measure perceived communication across the three most frequently used media in the office: face-to-face, phone, and email. Other media, such as instant messaging, Microsoft Teams, and Slack were not widely used by this design firm. The survey asked respondents to indicate how frequently they communicated with each studio team member over the past week, using a 5-point frequency scale (eg, never, once a week, two to three times a week, once per day, multiple times per day). For example: "Over the past week, how often did you exchange emails with this 


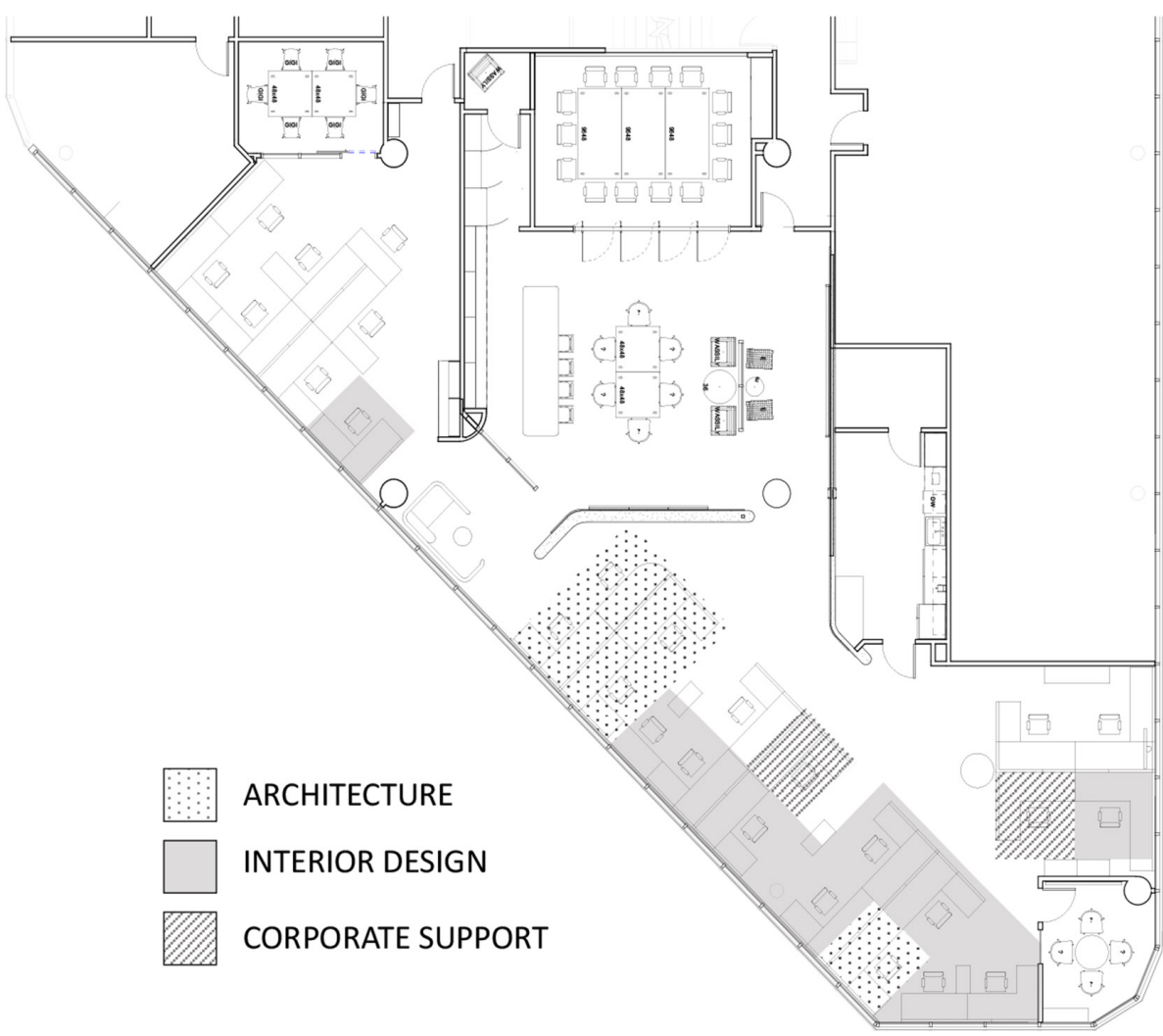

Figure 1 Layout of A/E design studio office with the location of team members by discipline.

person?" These questions did not ask who initiated the communication and were only measuring its frequency. The names of all studio team members appeared on the survey, and respondents could not skip or omit any name. Respondents were also asked to provide demographic information on themselves, including their gender, age, education, design discipline, tenure within the firm, and years of experience in the industry. There was only one minority race member of the sampled team. While race may be a relevant attribute for homophily, without any variation in the attribute, we were not able to include this attribute in the analysis.

\section{Data Collection Process}

The survey was distributed to the studio team through email. Each team member was asked to complete the survey about their communication during the weeks ending on July 19, 2019 and August 9, 2019. The surveys remained open for two weeks and obtained a response rate of $94 \%(n=17)$ and $100 \%(n=18)$ for each time period, respectively. These study weeks reflected a typical workweek
(Monday through Friday) under different degrees of time pressure. During the first period, work tasks focused on continuous production work and limited client meetings. This period represented a low time pressure work environment for the studio team. Team members were focused on producing construction documents detailing small interior design buildouts, referred to as tenant improvement projects, such as updating wall locations, flooring material, ceilings details, lighting types, and furniture selections. Team members would be expected to coordinate construction drawings between architects and interior designers within their studio team to complete this work. Because of the lack of project deadlines during this period, we suspect that individuals may have been more likely to communicate based more on their preferences rather than a formalised reporting hierarchy. During the second period, work tasks focused on producing design deliverables ahead of looming project deadlines, in addition to the more routine work tasks still being completed the first period. Because of the project deadlines occurring 
during this period, we suspect that individuals may have been more likely to communicate based on discipline and formalised reporting hierarchy to complete their required work tasks. No unique activities or events took place during either time periods, nor were there any recurring cycles of non-project related work within the organisation (eg, billing and collection).

\section{Dependent Variables}

The survey response data was organised into adjacency matrices that defined the topology of each communication network. The nodes in the network were the individual design team members. The perceived communication frequencies were used to denote the strength of ties among those members in the networks. Communication networks based on synchronous and bidirectional communication that occurred in real-time (eg, face-to-face and phone) were considered undirected in the analysis. In contrast, asynchronous and unidirectional communication (eg, emails) was considered directed. The resulting three communication networks are the dependent variables in the network analysis that will be predicted by demographic attribute differences in the studio team.

\section{Independent Variables}

There are six node-level attribute matrices that were created based on demographic data, including

Table 1 Node-level attribute matrices to predict communication

\begin{tabular}{|l|l|l|}
\hline $\begin{array}{l}\text { Node } \\
\text { attribute }\end{array}$ & Measure & Matrix type \\
\hline Gender & Yes (1); No (0) & Symmetric \\
\hline Discipline & Yes (1); No (0) & Symmetric \\
\hline Generation & Yes (1); No (0) & Symmetric \\
\hline $\begin{array}{l}\text { Age } \\
\text { difference }\end{array}$ & $\begin{array}{l}\text { The numerical } \\
\text { difference in age }\end{array}$ & Symmetric \\
\hline $\begin{array}{l}\text { Walk } \\
\text { distance }\end{array}$ & $\begin{array}{l}\text { Numerical distance } \\
\text { between workstations } \\
\text { (in feet) }\end{array}$ & Symmetric \\
\hline Hierarchy & Yes (1); No (0) & Asymmetric \\
\hline
\end{tabular}

gender, current age, generation, design discipline, hierarchy, and walk distance (see Table 1). These attribute matrices were created based on the difference between each pair of individuals, or dyad, in the studio team. The gender, generation, and discipline matrices were dichotomously coded using either a 1, when the dyad shared a common attribute or a 0 , when they differed in that attribute. The age difference and walking distance matrices were formed by subtracting the corresponding attribute (ie, numerical age or shortest physical distance in feet between workstations) for each dyad. Lastly, to capture information exchanges resulting from their current project's reporting structure, a hierarchy matrix was created. The hierarchy matrix was dichotomously coded using either a 1, when an individual reported directly to another individual, and a 0 otherwise. For example, an interior designer reports directly to a project manager. This relationship would be reflected as a one from the perspective of the interior design to project manager in the hierarchy matrix. However, this matrix was asymmetric. While the interior designer reports to the project manager, the project manager does not report to the interior designer. Thus, from the perspective of the project manager to the interior designer, the relationship would be reflected as a 0 in the hierarchy matrix. These six node-level matrices are the independent variables used to predict the studio team's communication. Demographic information on the respondent's tenure within the firm, years of experience in the industry, and education were collected during the study, but not used in the further analysis, as there was high conceptual overlap with other attributes. Specifically, tenure and experience were similar in practice to age and generation, while education was already captured by discipline. In the sampled firm, all practicing and licensed architects were required to have a master's degree and all interior designers were required to have a bachelor's degree or higher.

\section{Network Analysis}

Descriptive measures for each communication network were calculated using UCINET and visualisations were generated with NodeXL. These measures included density, average degree, and centralization. Density refers to the average strength 
between ties, which is measured through all total tie values divided by the total number of possible ties. The average degree identifies the average number of ties per node. These two measures distinguish the connectedness of the overall network with values signifying the level of connection within the network. The centralization index measures the extent to which ties are distributed throughout the network. Additionally, using UCINET, the data analysis was run with a multiple regression procedure (MR-QAP) to determine if similarities in participant backgrounds were significant predictors of their information exchanges for each communication medium.

Since homophily often involves a choice, either consciously or unconsciously, this analysis must control for other factors that may influence the decision with whom to communicate. Thus, the reporting structure of each participant to their co-workers and the physical distance between participants were used as controls in the regression to account for the effect of formalised information exchanges based on existing project hierarchies and proximity effecting the ease of communication, respectively. The reporting structure represents the formal communication within the organisation and information must flow along these channels as a matter of policy or procedure within the organisation. Likewise, prior research has consistently found that individuals close to one another in physical distance are highly likely to interact, especially in face-to-face communication.
Both these formal channels and proximity-based communication are not seen as a choice. By including them in the analysis, we can determine with greater certainty whether team member attributes are still predictive of any communication when hierarchy and proximity are also considered.

\section{RESULTS}

The results are separated in four sections. First, we present participant demographics, which show the composition of the design studio team to provide context for subsequent results. Second, we report network correlations between the communication and attribute networks. Next, we present the results of the network regression analysis to determine which node attribute similarities are predictive of each communication network. Lastly, we provide network-level measures by communication medium for low and high time pressure work environments, including presentation of relevant network visualisations.

\section{Participants Demographics}

A total of 17 studio team members responded to the first survey in the low time pressure environment. A summary of the demographics of these respondents is provided in Table 2. The ages of respondents ranged from 24 to 62 years of age. The largest age bracket contained $7(41 \%)$ team members

Table 2 Participant demographics at low time pressure

\begin{tabular}{|l|r|l|l||l|l|}
\hline & $\boldsymbol{N}$ & $\mathbf{\%}$ & & $\boldsymbol{N}$ & $\%$ \\
\hline Gender & & & Generation & & \\
\hline Male & 5 & $29 \%$ & Millennial & 7 & $41 \%$ \\
\hline \hline Female & 12 & $71 \%$ & Generation X & 9 & $53 \%$ \\
\hline & & & Baby Boomer & 1 & $6 \%$ \\
\hline Age (years) & & & & & \\
\hline $20-29$ & 4 & $23 \%$ & Discipline & & \\
\hline $30-39$ & 3 & $18 \%$ & Architecture & 7 & $41 \%$ \\
\hline $40-49$ & 7 & $41 \%$ & Interior Design & 8 & $47 \%$ \\
\hline $50-59$ & 2 & $12 \%$ & Corporate Support & 2 & $12 \%$ \\
\hline 60 or over & 1 & $6 \%$ & & & \\
\hline
\end{tabular}

Engineering Project Organization Journal

(C) 2021 Engineering Project Organization Society www.epossociety.org 
Table 3 Correlation matrix for networks at low time pressure

\begin{tabular}{|l|r|r|r|r|r|r|r|l|}
\hline & \multicolumn{1}{l}{} & \multicolumn{1}{l}{ 2. } & $\mathbf{3 .}$ & $\mathbf{4}$ & $\mathbf{5 .}$ & $\mathbf{6 .}$ & $\mathbf{7 .}$ & $\mathbf{8 .}$ \\
\hline 1. Face-to-face & & & & & & & & \\
\hline 2. Phone & $* 0.20$ & & & & & & & \\
\hline 3. Email & $* * 0.36$ & $* * * 0.48$ & & & & & & \\
\hline 4. Gender & $* * 0.30$ & 0.01 & -0.04 & & & & & \\
\hline 5. Age difference & -0.04 & 0.12 & 0.13 & -0.14 & & & & \\
\hline 6. Generation & 0.02 & 0.04 & -0.00 & 0.02 & $* * *-0.70$ & & & \\
\hline 7. Discipline & 0.16 & $* 0.15$ & 0.09 & $* * 0.36$ & -0.05 & 0.00 & & \\
\hline 8. Hierarchy & 0.13 & $* * * 0.29$ & $* * * 0.29$ & 0.11 & 0.00 & -0.03 & $* 0.20$ & \\
\hline 9. Walk distance & $* * *-0.48$ & 0.04 & -0.13 & -0.07 & -0.07 & 0.05 & 0.03 & $*-0.17$ \\
\hline
\end{tabular}

$* P<0.05, * * P<0.01, * * * P<0.001$.

between 40-49 years of age and was followed by $4(23 \%)$ between the years of age $20-29,3(18 \%)$ between 30-39 years of age, 2 (12\%) between 50-59 years of age, and $1(6 \%)$ over the age of 60 . By generation, Generation X (birth year between 1961-1981) had the largest representation with 9 (53\%) respondents, Millennials (1982-2004) with 7 (41\%), and Baby Boomers (1943-1960) with $1(6 \%)$. The sample studio team primarily represented those with architecture (41\%) and interior design (47\%) background but also included $2(12 \%)$ corporate support members.

During the second data collection in the high time pressure environment, an additional team member was included $(n=18)$. The new team member was hired the week following the first data collection and became an integral part of the team for three weeks before the time of the second data collection. The new team member was a female, 20-29 years of age, a member of the Millennial generation, within the interior design practice, and in the role of designer.

\section{Network Correlations}

To examine the relationship between similarities in the studio team's attributes and their communication patterns, a QAP correlation was performed for both low time pressure (LTP) and high time pressure (HTP) environments. The resulting correlations are summarised in Tables 3 and 4. The correlated networks include the three communication

Table 4 Correlation matrix for networks at high time pressure

\begin{tabular}{|l|r|r|r|r|r|r|r|l|}
\hline & \multicolumn{1}{|l|}{} & $\mathbf{2 .}$ & $\mathbf{3 .}$ & $\mathbf{4 .}$ & $\mathbf{5 .}$ & $\mathbf{6 .}$ & 7. & 8. \\
\hline 1. Face-to-face & & & & & & & & \\
\hline 2. Phone & $* * * 0.52$ & & & & & & & \\
\hline 3. Email & $* * * 0.59$ & $* * * 0.63$ & & & & & & \\
\hline 4. Gender & 0.06 & 0.05 & 0.04 & & & & & \\
\hline 5. Age difference & 0.02 & 0.06 & 0.06 & 0.08 & & & & \\
\hline 6. Generation & 0.03 & 0.06 & 0.02 & -0.02 & $* * *-0.71$ & & & \\
\hline 7. Discipline & $* 0.21$ & $* 0.16$ & 0.07 & 0.07 & -0.06 & 0.01 & & \\
\hline 8. Hierarchy & $* * * 0.35$ & $* * * 0.38$ & $* * * 0.40$ & -0.01 & 0.01 & -0.05 & $* 0.19$ & \\
\hline 9. Walk distance & $* *-0.34$ & -0.03 & $*-0.20$ & -0.06 & -0.03 & 0.01 & 0.02 & $*-0.16$ \\
\hline
\end{tabular}

$* P<0.05, * * P<0.01, * * * P<0.001$. 
Table 5 Communication network regressions results at low time pressure

\begin{tabular}{|l|c|l|l|c|}
\hline Predictor & Face-to-Face & Phone & Email & Combined \\
\hline Constant & $* * * 0.00$ & $* * * 0.00$ & $* * * 0.00$ & $* * * 0.00$ \\
\hline Gender & $* * 0.23$ & -0.01 & -0.09 & 0.09 \\
\hline Age difference & -0.00 & $* 0.31$ & 0.25 & 0.21 \\
\hline Generation & 0.06 & $* * * 0.29$ & $*-0.19$ & $* 0.19$ \\
\hline Generation & 0.09 & 0.12 & 0.09 & 0.11 \\
\hline Hierarchy & 0.01 & $* * * 0.30$ & $* * * 0.27$ & $* * 0.21$ \\
\hline Walk distance & $* * *-0.48$ & 0.10 & -0.07 & $*-0.27$ \\
\hline$R 2$ & $32.25 \%$ & $16.10 \%$ & $13.62 \%$ & $19.95 \%$ \\
\hline
\end{tabular}

${ }^{*} P<0.05,{ }^{*} P<<0.01, * * * P<0.001$

media (face-to-face, phone, email), gender, age difference, generation, discipline, hierarchy, and walk distance. There were significant $(P<0.05)$ correlations between face-to-face and phone communication networks $\left(\mathrm{r}_{\mathrm{LTP}}=0.20, \mathrm{r}_{\mathrm{HTP}}=0.52\right)$, between face-to-face and email communication $\left(r_{\text {LTP }}=0.36, r_{\text {HTP }}=0.59\right)$, and between face-to-face communication and walk distance $\left(\mathrm{r}_{\mathrm{LTP}}=-0.48\right.$, $\left.r_{\text {HTP }}=-0.34\right)$. These relationships suggest that multiple forms of communication are being used between team member dyads, such that an increase in communication is one medium is met with an increase in another medium. The attribute walk distance expresses the physical distance in feet, rounded to the nearest whole number, between two individuals. Thus, a negative correlation between face-to-face communication and walk distance means that as the distance increases between individuals, there is a decrease in face-toface communication. There was also a significant correlationbetween phoneandemail communication $\left(\mathrm{r}_{\text {LTP }}=0.48, \quad \mathrm{r}_{\mathrm{HTP}}=0.63\right)$, phone communication and discipline $\left(\mathrm{r}_{\mathrm{LTP}}=0.15, \mathrm{r}_{\mathrm{HTP}}=0.16\right)$, and phone communication and hierarchy $\left(\mathrm{r}_{\mathrm{LTP}}=0.29, r=0.38\right)$. Lastly, there was a significant correlation between email communication and hierarchy $\left(\mathrm{r}_{\mathrm{LTP}}=0.29\right.$, $\left.\mathrm{r}_{\mathrm{HTP}}=0.38\right)$. In terms of individual attributes, generation was highly negatively correlated with age difference $\left(\mathrm{r}_{\text {LTP }}=-0.70, \mathrm{r}_{\text {HTP }}=-0.71\right)$, discipline was positively correlated with hierarchy $\left(\mathrm{r}_{\text {LTP }}=0.20\right.$, $\mathrm{r}_{\mathrm{HTP}}=0.19$ ), and walk distance was negatively correlated with hierarchy $\left(\mathrm{r}_{\text {LTP }}=-0.17, \mathrm{r}_{\mathrm{HTP}}=-0.17\right)$.

Despite the many similarities between the low and the high time pressure periods, there was one notable difference. Gender was significantly correlated with face-to-face communication in the

Table 6 Communication network regressions results at high time pressure

\begin{tabular}{|l|c|l|l|c|}
\hline Predictor & Face-to-Face & Phone & Email & Combined \\
\hline Constant & $* * * 0.00$ & $* * * 0.00$ & $* * * 0.00$ & $* * * 0.00$ \\
\hline Gender & 0.10 & -0.01 & -0.08 & 0.01 \\
\hline Age difference & 0.14 & 0.24 & 0.16 & 0.20 \\
\hline Generation & 0.15 & $* * 0.25$ & 0.16 & $* 0.21$ \\
\hline Discipline & $* 0.14$ & 0.10 & 0.04 & 0.10 \\
\hline Hierarchy & $* * * 0.28$ & $* * * 0.38$ & $* * * 0.38$ & $* * * 0.40$ \\
\hline Walk distance & $* * *-0.29$ & 0.03 & -0.15 & $*-0.18$ \\
\hline$R 2$ & $25.45 \%$ & $19.49 \%$ & $20.19 \%$ & $25.79 \%$ \\
\hline
\end{tabular}

$* P<0.05,{ }^{* *} P<0.01,{ }^{* * *} P<0.001$. 
low time pressure environment $\left(\mathrm{r}_{L T P}=0.30\right)$, but not high time pressure. Instead, in the high time pressure environment, there was a significant correlation between hierarchy and face-to-face communication $\left(\mathrm{r}_{H T P}=0.35\right)$ that was not present in the low time pressure period.

\section{Network Regression}

Using UCINET, a multiple regression (MR-QAP) was performed for each communication network to determine if individual similarities predicted the network topology. The results of these regressions are summarised in Tables 5 and 6 . While there were differences across communication media, and while the percentage of variance explained $\left(R^{2}\right)$ ranged from only $13 \%$ to $32 \%$, the regression analysis provided evidence that homophily contributed to the structure of the networks when under low time pressure. Specifically, faceto-face conversations were more likely when designers were within a shorter walking distance of one another $\left(\beta_{L T P}=-0.48, P=0.00\right)$. Face-toface conversations were more likely when the exchange took place between the same gender $\left(\beta_{L T P}=0.23, P=0.00\right)$. Phone conversations were more likely when designers were from the same generation $\left(\beta_{L T P}=0.29, P=0.00\right)$ and along reporting ties explained by the hierarchy of the studio team $\left(\beta_{L T P}=0.30, P=0.00\right)$. Email exchanges were more likely when the designers were from different generations $\left(\beta_{L T P}=-0.19, P=0.05\right)$ and were only explained by the hierarchy of the studio team $\left(\beta_{L T P}=0.27, P=0.00\right)$.

In addition to the communication networks by medium, a fourth regression was performed on the overall communication occurring within the design studio team during each time of data collection. This combined network was created by adding all communication frequencies from the three media and reflects a rough approximation of the total communication occurring within the study period. For the low time pressure period, communication in this combined network was predicted by generation $\left(\beta_{L T P}=0.19, P=0.05\right)$, hierarchy $\left(\beta_{L T P}=0.21, P=0.00\right)$, and walk distance $\left(\beta_{L T P}=-0.27, P=0.03\right)$, suggesting that team members within the same generation to one another may prefer certain communication media.
During the high time pressure period, there were also differences across communication media. The percentage of variance explained ranged from 19\% to $35 \%$, again providing evidence that homophily contributed to the structure of the networks. During this period, face-to-face conversations were explained by discipline $\left(\beta_{H T P}=0.14, P=0.05\right)$, the hierarchy of the studio team $\left(\beta_{\text {HTP }}=0.28, P=0.00\right)$, and walk distance $\left(\beta_{H T P}=-0.29, P=0.00\right)$. Phone conversations were more likely when designers were from the same generation $\left(\beta_{H T P}=0.25, P=0.01\right)$ and along hierarchical ties found in the reporting structure of the studio team $\left(\beta_{\text {HTP }}=0.39, P=0.00\right)$. Email exchanges were only explained by the team hierarchy $\left(\beta_{H T P}=0.40, P=0.00\right)$, and therefore there is little evidence that homophily was present in email exchanges.

Again, a fourth regression was performed on the overall communication occurring within the design studio team during the high time pressure period. In this regression, communication in this combined network was predicted by generation $\left(\beta_{H T P}=0.21, \quad P=0.05\right), \quad$ hierarchy $\quad\left(\beta_{H T P}=0.40\right.$, $P=0.00)$, and walk distance $\left(\beta_{H T P}=-0.18, P=0.03\right)$ once again suggesting that team members within the same generation prefer certain communication media. Notably, the same set of attributes predicted the overall communication in both low and high time pressure work environments.

\section{Network Description}

Under low time pressure, the face-to-face communication network had a density of 0.467 , an average degree of 7.471, and a centralization index of 0.242 (see Table 7). The phone communication network had a density of 0.140 , an average degree of 2.235, and a centralization index of 0.196 . The email communication network had a density of 0.607, an average degree of 9.706, and a centralization index of 0.485 . During the high time pressure, in terms of whole network measures, the face-to-face communication network in the second data collection had a density of 0.484 , an average degree of 8.222 , and a centralization index of 0.219 . The phone communication network had a density of 0.203 , an average degree of 3.444 , and a centralization index of 0.195 . The email communication network had a density of 0.680 , 
Table 7 Network-level measures by medium and time pressure

\begin{tabular}{|c|c|c|c|}
\hline & \multicolumn{3}{|c|}{ Communication network } \\
\hline & $\begin{array}{l}\text { Face-to- } \\
\text { Face }\end{array}$ & Email & Phone \\
\hline \multicolumn{4}{|l|}{$\begin{array}{l}\text { Low Time } \\
\text { Pressure }\end{array}$} \\
\hline Density & 0.467 & 0.607 & 0.140 \\
\hline Centralization & 0.242 & 0.485 & 0.196 \\
\hline Average Degree & 7.147 & 9.706 & 3.235 \\
\hline \multicolumn{4}{|l|}{$\begin{array}{l}\text { High Time } \\
\text { Pressure }\end{array}$} \\
\hline Density & 0.484 & 0.680 & 0.203 \\
\hline Centralization & 0.219 & 0.397 & 0.195 \\
\hline Average Degree & 8.222 & 11.556 & 3.444 \\
\hline \multicolumn{4}{|l|}{$\begin{array}{l}\text { \% Change (Low } \\
\text { to High) }\end{array}$} \\
\hline Density & $3.6 \%$ & $12.0 \%$ & $45.0 \%$ \\
\hline Centralization & $-9.5 \%$ & $-18.1 \%$ & $-0.5 \%$ \\
\hline Average Degree & $15.0 \%$ & $19.1 \%$ & $6.5 \%$ \\
\hline
\end{tabular}

an average degree of 11.556 , and a centralization index of 0.397 .

As compared in Table 7, most network-level measures remain similar within medium and across time pressures. Across both low and high time pressures, the email communication network was the most connected by average degree, followed by the face-to-face network. The least used communication medium was phone in both time periods. Of the three communication networks, phone and face-to-face were the least effected by time pressure. For email communication, however, the density and average degree increase significantly when moving from a low time pressure to high time pressure environment. Notably, all three media see a reduction in centralization under high time pressure.

Network graphs were created to illustrate selected homophilous relationships identified through the regression analysis (see Figure 2). The network visualisations were prepared using NodeXL, using a circular graphing function with groups created for each attribute of interest. In Figure 2, the communication networks for faceto-face and phone as shown, as these networks showed evidence of homophily in the regression analysis. The ties connecting each node signify a strength of either multiple interactions per day in the case of face-to-face communication, or multiple interactions per week, in the case of phone communication. These frequency cutoffs for tie display were selected to simplify the graphs and to make the relationships between attributes and frequent communication more apparent.

During the low time pressure period, the faceto-face communication network shows a greater number of ties between the same gender, when compared to the same team during the high time pressure period (Figure 2a). During the high time pressure period, similarity in discipline became the primary predictor of face-to-face interactions, which was not present in the low time pressure period (Figure 2b). Lastly, similarity in generation was a significant predictor of phone communication, regardless of the time pressure experienced by the studio team (Figure 2c). Additionally, all networks were highly centralised around the nodes identified as $\mathrm{A}, \mathrm{B}$, and $\mathrm{C}$ during both time periods. Node A is the lead licensed architect who reviews drawings and signs and seals documents for the permitting process. This role requires interactions between architecture, design, and administrative teams. Node B is an interior designer who was acting in a junior project manager role at the time of the study, which requires coordinating internal support and production staff. Node $\mathrm{C}$ is the workplace studio principal. While his background is in architecture, his role entails directing the overall studio staffing of projects, project demand, lead for planning, managing, monitoring, and coordinating of both the architecture and interior design teams.

\section{DISCUSSION}

With the increasing complexity of building design, $\mathrm{A} / \mathrm{E}$ studio teams are expected to collaborate more frequently with other disciplines, often under intense time pressure. This collaboration occurs through a growing number of communication media, such as face-to-face conversations, phone calls, and emails. Various media theories attempt to explain how individuals choose among these media, based on the type and quantity of information being 


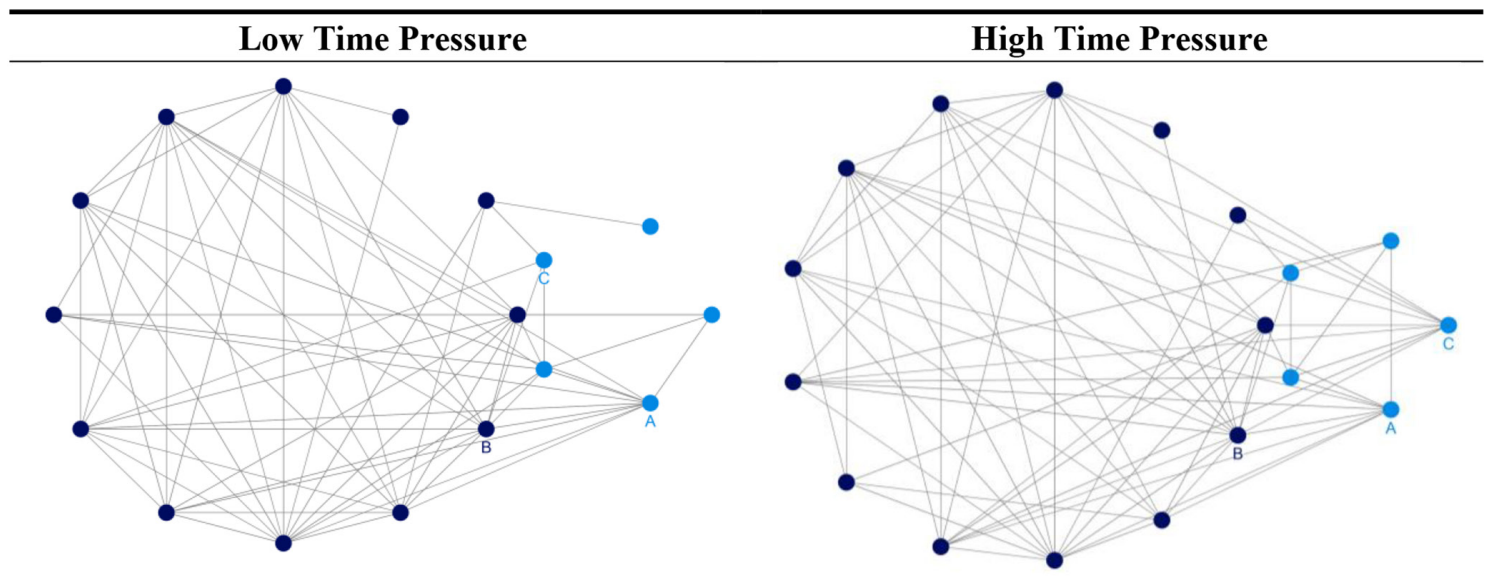

a. Reported face-to-face communication occuring multiple times each day, showing homophily by gender ( $\bullet$ denotes a female team member, while $\bullet$ denotes male) only in low time pressure work environments
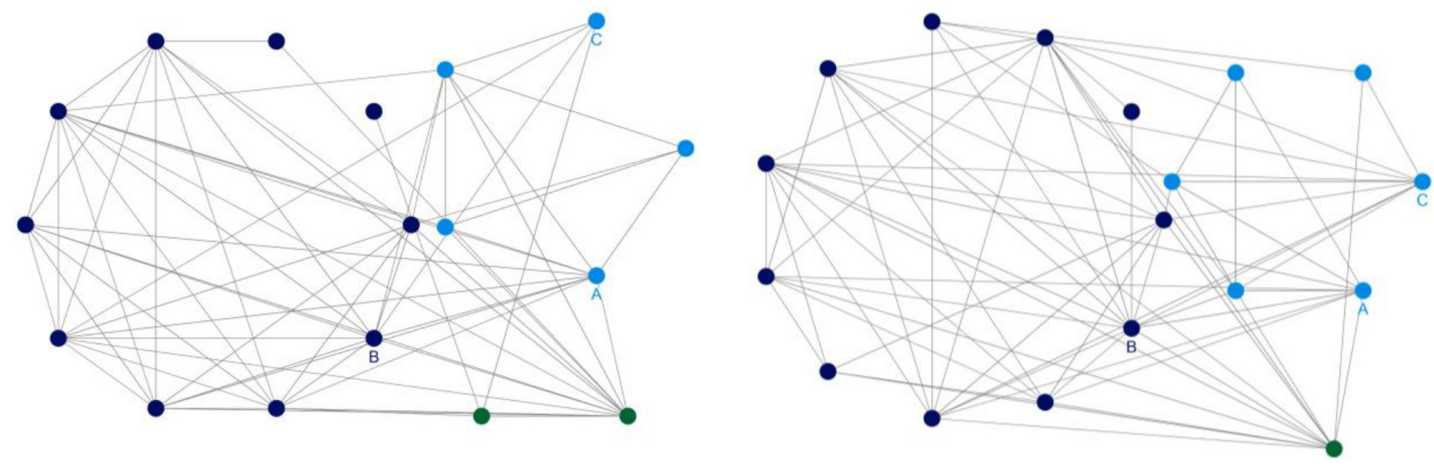

b. Reported face-to-face communication occuring multiple times each day, showing homophily by discipline (• denotes interior design expertise, $\bullet$ denotes architecture, and $\bullet$ denotes adminstrative) only in high time pressure work environments
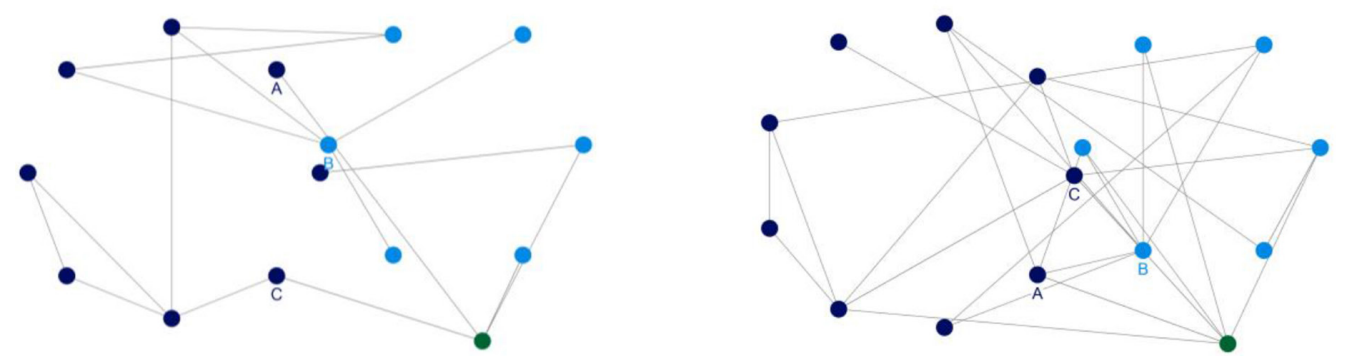

c. Reported phone communication occuring multiple times per week, showing greater homophily by generation (• denotes interior Generation X, $\bullet$ denotes Millennial, and $\bullet$ denotes Baby Boomer) in both low and high time pressure work environments

Figure 2 Communication network graphs by medium and time pressure.

conveyed (Daft et al., 1987; Dennis and Kinney, 1998). In addition to the medium, individuals also choose the people with whom they communicate and how frequently. When the work environment is centralised or hierarchical, people communicate primarily along formal channels by receiving 
Table 8 Comparison of homophily under low and high time pressure work environments

\begin{tabular}{|l|l|l|}
\hline \multicolumn{2}{|l|}{} & Work environment \\
\hline Communication Network & Low time pressure & High time pressure \\
\hline Face-to-Face & Gender $\left(0.23^{* *}\right)$ & Discipline $\left(0.14^{*}\right)$ \\
\hline Phone & Age $\left(0.31^{*}\right)$, Generation $\left(0.29^{* * *}\right)$ & Generation $\left(0.25^{* *}\right)$ \\
\hline Email & Generation $\left(-0.19^{*}\right)$ & - \\
\hline Combined & Generation $\left(0.19^{*}\right)$ & Generation $\left(0.21^{*}\right)$ \\
\hline
\end{tabular}

$* P<0.05,{ }^{*} P<0.01, * * * P<0.001$.

information from their subordinates and passing information to their supervisor (Bromley et al., 2003; Love et al., 1998; Moore and Dainty, 2001). However, in more decentralised or networked environments such as those found in studio design teams, people have more freedom in deciding how and with whom they communicate (Bromley et al., 2003). This study explored the extent to which this freedom of association, in a surveyed $\mathrm{A} / \mathrm{E}$ studio team, resulted in homophilous networks under low and high time pressure work environments. Homophilous networks are widely viewed as detrimental for creative tasks.

The results suggest that homophily was present to a limited degree in the face-to-face, phone, and email communication networks, when controlling for the studio team's hierarchical reporting structure and physical distance from one another in the office (see Table 8). In the studied studio team, structural homophily was expected because of the unequal proportion of men and women in the composition of the team. The team was $71 \%$ female, meaning that simply by chance, more female-to-female communication ties are expected (Ibarra, 1992; Leenders, 1996; McPherson et al., 2001). That expectation should extend to all communication media, including face-to-face, phone, and email. However, our findings did not provide evidence of structural gender homophily. Team members exhibited a preference for communicating with the same gender only in face-to-face interaction and only when not under intense deadline pressure. This finding is consistent with literature reporting that individuals will often group themselves based on gender (Bielby and Baron, 1986; Kalleberg et al., 1996; McPherson and Smith-Lovin, 1982, p. 1986; South et al., 1982; South et al., 1983).
However, the apparent moderating effect of time pressure on gender homophily in face-to-face interaction suggests that this relationship is only prevalent in low pressure work environments, where team members communication may be driven more by preference than need. When under high time pressure to complete design deliverables, individuals were incentivised, either formally or informally, to communicate with those in the same discipline that could likely assist them in completing their tasks.

Despite a lack of structural homophily, choice homophily was reflected within both phone and email communication networks. While phone and email exchanges are facilitated by technology, these media differ in their synchronicity. Synchronous communication means exchanging information in real-time and often rapidly (eg, face-to-face or phone). Asynchronous exchanges do not require an immediate response and allow individuals more time to consider the conversation carefully (eg, email or text messages). The network regression results show that studio team members from the same generation were more likely to communicate synchronously by phone during both time pressure periods. In contrast, members from different generations were more likely to communicate asynchronously through email. This difference could be interpreted as evidence of choice homophily by generation and is pervasive in both low and high time pressure environments. However, the homophily observed may be incidental to a generational preference for one medium over another. That is, each generation having lived through an era dominated by a particular medium may continue to exhibit a preference for that medium, despite a growing number of ways 
to communicate. As a result, they may choose to communicate with others having the same preference, who incidentally are more likely to be from the same generation. Documented differences in generations provide support for this explanation. In general, the Baby Boomer generation prefers formal and structured communication, such as phone calls, in-person conversations, organised meetings, and scheduled appointments. Generation $\mathrm{X}$ and Millennials, on the other hand, prefer informal, less layered communication styles through emails and text messages (Wagner, 2007). These informal media allow members of both Generation X and Millennials to multitask and maintain numerous ongoing dialogues, making them flexible and resourceful communicators and permitting communication to occur continuously (Gale, 2007). More importantly, these exchanges are asynchronous and allow members of the younger generation time to formulate responses, without being put "on the spot" in every interaction and information exchange.

While there is evidence of moderation by time pressure within the communication networks of certain media, time pressure does not appear to have the same effect when comparing the total communication occurring across networks. In both the low and high time pressure periods, only generational similarity predicted the combined communication network topology, when controlling for both reporting structure and walking distance between team members. This strongly suggests that shared sub-cultures within generations, comprised of team members at the same or similar stages in their life, are a persistent source of homophily in the sampled A/E studio team.

In addition to understanding the extent of homophily within an $\mathrm{A} / \mathrm{E}$ studio team during a low and high time pressure, we also sought to identify ways to reduce or limit homophilous communication. Counterintuitively, the results suggest that a shorter walking distance between team members reduced choice homophily within the studied workplace for face-to-face communication. In other words, team members that were physically closer to one another were more likely to engage in frequent face-to-face interaction, regardless of their differences in age, generation, or discipline.
However, this varied based on the level of time pressure. Prior research found that spatial location did not determine a tie but rather the strength of a relationship (McPherson et al., 2001). Additional research will need to be conducted to conclude the strength of a relationship under differencing time pressure environments, as Chong et al. (2012) found those under periods of high time pressure did not benefit from close proximity.

This study's finding may have implications in designing workplaces and individual placement within that environment. Members of the sampled A/E studio team were seated in their workplace according to their active projects and individual members did not select their workstation. They were assigned by the office president and studio leadership, and not based on gender, age, or discipline, but rather their closeness to other team members working on the same or similar projects. There was no rearrangement of team members based on schedule deadlines. Thus, there is evidence that organising the workplace by a project and placing all the studio team members near one another reduced homophily in the faceto-face communication network. However, neither the data nor the findings can determine whether the proximity of team members would reduce choice homophily if a studio team were grouped based on their background, discipline, or general role instead of the project. The effectiveness of limiting homophily through workplace design and team placement requires additional exploration. Proximity needs to be revisited, as decreased physical distance increases the ease of communication under select time pressure periods, regardless of the similarity of those exchanging the information. However, if workplaces are seated by discipline, individuals would be required to travel further to reach a diverse opinion.

\section{Limitations}

While examining homophily in the sampled studio team provided some insight into how designers communicate, there are several limitations to acknowledge. First, the findings from a single team cannot be generalised directly to all workplaces due to each organisation's unique ethos, the distinct personalities of the employees, communication 
styles, and the workplace culture. The research location was selected, in large part, because of the availability and willingness of the site to participate in the study. The researcher conducted this single case study over two months and limited the sample data to a modest size $(n=17, n=18)$. However, this design studio is representative of large architecture, design, and engineering organisations in the United States. Thus, the findings provide a starting point for $\mathrm{A} / \mathrm{E}$ organisations seeking to improve collaboration in their workplace. Second, the data was collected for two work weeks (ie, low and high time pressure environments) and future work may consider controlling for the tasks being performed when evaluating the topology of the network. Third, this research does not relate diverse team member backgrounds directly to better project outcomes or design solutions. Prior literature makes clear the link between diversity of ideas and better team outcomes; however, the extent to which homophily of gender and generation effect team outcomes is still largely unexplored. Lastly, data was collected based on the team member's perception of their communication frequency and not physical records of their communication (eg, number of emails sent and received). This study assumed that employees offered honest and accurate responses when completing the surveys. However, there is the potential for error as respondents may exhibit bias, either from poor memory or by providing a socially correct answer that differs from reality.

\section{CONCLUSIONS}

This study investigated the workplace interactions in an $\mathrm{A} / \mathrm{E}$ design studio team to determine the extent to which homophily is exhibited in their communication during periods of low and high time pressures. An online survey was distributed to collect data on the frequency of interactions among team members across a variety of communication media when comparing changes occurring during low and high time pressure. A social network regression analysis was performed to relate similarities in team member dyads with the structure of the communication networks. The results show some evidence of choice homophily as a predictor of interactions under low time pressure when controlling for the hierarchy of the studio team and their physical distance from one another. Specifically, face-to-face communication was more likely when members were of the same gender and under low time pressure, while phone communication was more likely only when members were from the same generation, regardless of the time pressure. For email communication, there was no evidence of homophily in any media or under any time pressure. In low time pressure work environments, team members from different generations were actually more likely to communicate over email and in high time pressure, the only consistent predictor of email communication was the team hierarchy.

The results of this study showed that changes occurring in the frequency of use of media and presence of homophily with communication media varied by the presence or absence of time pressure. This finding reveals the need for future research, as most time constraint studies can be generalised into two categories: studies dealing with the effects of time pressure on team process and structure, and those examining the effects of time pressure on group productivity (Bennett et al., 1973; Frye and Stritch, 1964; Pruitt and Drews, 1969; Pruitt and Johnson, 1970; Schutz, 1955). The gap within previous literature and studies reveals a potential for future research focus on how team member attributes influence their choice of communication medium when under time pressure, and the effect that choice has on the quality of the team's deliverables. This area of research may be increasingly relevant, as teams experiment with a greater number of virtual collaboration tools and as proximity increases because of social distancing and working-from-home, all lessons learnt during the COVID-19 era.

The ability to measure patterns of interactions will be meaningful in operationalizing the structure of project teams, planning deliverables and deadlines, fueling projects with differing ideas, allowing trust to form on teams, and informing the physical layout of workplaces. In addition to the potential influences on the organisation and workplace layout structure, this process can reveal the sociodemographic factors that impact the selection of communication media, building upon earlier theories rooted in richness, perception, and 
outcome. Within today's workplaces, diversity is abundant and complicated by the need for more significant multidisciplinary communication. Thus, this research has implications for the design of studio workplaces that support information-rich interactions, the assignment of individual designers to project teams that are more likely to interact with co-workers from different backgrounds, and organisational policy regarding the use of specific communication media based on the project schedule and time pressure.

\section{References}

Allen, T.J. (1970), "Communication networks R\&D laboratories", R\&D Management, Vol. 1 No. 1, pp. $14-21$.

Allen, T.J. (2007), "Architecture and communication in product development engineers", California Management Review, University of California, Vol. 49 No. 2, pp. 23-41.

Allen, T.J. and Henn, G.W. (2007), The organization and architecture of innovation: Managing the flow of technology. New York, NY: Taylor \& Francis.

Alwin, D.F. and McCammon, R.J. (2007), "Rethinking generations", Research in Human Development, Vol. 4 No. 3-4, pp. 219-237. 10.1080/15427600701663072

Argote, L. (1999), Organizational learning: creating, retaining, and transferring knowledge. Boston, MA: Kluwer Academic.

Argote, L. and Ophir, R. (2002), "Intraorganizational learning". In: Baum J. A. C, ed, Companion to Organizations. Malden, MA: Blackwell. pp. 181-207.

Argote, L., Turner, M.E. and Fichman, M. (1989), "To centralize or not to centralize: the effects of uncertainty and threat on group structure and performance", Organizational Behavior and Human Decision Processes, Vol. 43 No. 1, pp. 58-74. 10.1016/0749-5978(89)90058-7

Becker, F. (2004), Offices at work: Uncommon workspace strategies that add value and improve performance. San Francisco, CA: Jossey-Bass.

Bennett, C., Lindskold, S. and Bennett, R. (1973), "The effects of group size and discussion time on the risky shift", The Journal of Social Psychology, Vol. 91 No. 1, pp. 137-147. 10.1080/00224545.1973.9922655

Bielby, W.T. and Baron, J.N. (1986), "Men and women at work: sex segregation and statistical discrimina- tion”, American Journal of Sociology, Vol. 91 No. 4, pp. 759-799. 10.1086/228350

Brashears, M.E. (2008), "Gender and homophily: differences in male female association in Blau space", Social Science Research, Vol. 37 No. 2, pp. 400-415. 10.1016/j.ssresearch.2007.08.004

Brass, D.J. (1985), “Men's and women's networks: a study of interaction patterns and influence in an organization", Academy Management Journal, Vol. 28, pp. 327-343. https://doi.org/10.2307/256204

Brass, D.J., Galaskiewicz, J., Greve, H.R. and Tsai, W. (2004), "Taking stock of networks and organizations: a multilevel perspective", The Academy of Management Journal, Vol. 47, pp. 795-817. https:// doi.org/10.5465/20159624

Bromley, S., Worthington, J. and Robinson, C. (2003), The impact of integrated teams on the design process. London: Construction Productivity Network.

Brown, T.M. and Miller, C.E. (2000), "Communication networks in task-performing groups: effects of task complexity, time pressure and interpersonal dominance", Small Group Research, Vol. 31 No. 2, pp. 131-157. https://doi.org/10.1177/ 104649640003100201

Byrne, D.E. (1971), The Attraction Paradigm. New York: Academic Press.

Caballer, A., Gracia, F. and Peiro', J.M. (2005), “Affective responses to work process and outcomes in virtual teams. Effects of communication media and time pressure", Journal of Managerial Psychology, Vol. 20, pp. 245-260. https://doi.org/10.1108/ 02683940510589037

Charles, K.E. and Veitch, J.A. (2002), Environmental satisfaction in open-plan environments: 2. Effects of workstation size, partition height and windows (IRC-IR-845). Ottawa, Ontario: National Research Council Canada, Institute for Research in Construction.

Chong, D.S.F., Eerde, W., Rutte, C.G. and Chai, K.H. (2012), "Bringing employees closer: the effect of proximity on communication when teams function under time pressure", Journal of Product Innovation Management, Vol. 29 No. 2, pp. 205-215. 10.1111/j.1540-5885.2011.00890.x

Chu, P.C. and Spires, E.E. (2001), "Does time constraint on users negate the efficacy of decision support systems?" Organizational Behavior and Human Decision Processes, Vol. 85 No. 2, pp. 226-249. 10.1006/obhd.2000.2940

Daft, R.L., Lengel, R.H. and Trevino, L.K. (1987), "Message Equivocality, media selection, and manager performance: implications for information sys-

Engineering Project Organization Journal

(C) 2021 Engineering Project Organization Society www.epossociety.org 
tems", MIS Quarterly, Vol. 11 No. 3, pp. 355-366. $10.2307 / 248682$

Dennis, A.R. and Kinney, S.T. (1998), “Testing media richness theory in the new media: the effects of cues, feedback, and task equivocality", Information Systems Research, Vol. 9 No. 3, pp. 256-274. 10.1287/isre.9.3.256

Feld, S. (1982), "Structural determinants of similarity among associates", American Sociology Review, Vol. 47, pp. 797-801.

Fischer, C.S. (1977), Networks and Places: Social Relations in the Urban Setting. New York: Free Press.

Fischer, C.S. (1982), To dwell among friends. Chicago: University of Chicago Press.

Fried, Y., Slowik, L.H., Ben-David, H.A. and Tiegs, R.B. (2001), "Exploring the relationship between workspace density and employee attitudinal reactions: an integrative model", Journal of Occupational and Organizational Psychology, Vol. 74 No. 3, pp. 359-372. 10.1348/096317901167406

Frye, R.L. and Stritch, T.M. (1964), "Effect of timed vs. NONTIMED discussion upon measures of influence and change in small groups", The Journal of Social Psychology, Vol. 63 No. 1, pp. 139-143. 10.1080/00224545.1964.9922221

Gale, S.F. (2007), "Bridging the gap", PM Network, Vol. 21, pp. 26-31.

Ibarra, H. (1992), "Homophily and differential returns: sex differences in network structure and access in an advertising firm", Administrative Science Quarterly, Vol. 37 No. 3, pp. 422-447. 10.2307/2393451

Isenberg, D.J. (1981), "Some effects of time-pressure on vertical structure and decision-making accuracy in small groups", Organizational Behavior and Human Performance, Vol. 27 No. 1, pp. 119-134. 10.1016/0030-5073(81)90042-8

Janis, I.L. (1972), Victims of Groupthink: A psychological study of foreign-policy decisions and fiascoes. Boston, MA: Houghton Mifflin.

Kalleberg, A.L., Knoke, D., Marsden, P.V. and Spaeth, J.L. (1996), Organizations in America: Analyzing their structures and human resource practices. Thousand Oaks CA: Sage.

Kelly, J.R. and McGrath, J.E. (1985), "Effects of time limits and task types on task performance and interaction of four-person groups", Journal of Personality and Social Psychology, Vol. 49 No. 2, pp. 395-407. 10.1037/0022-3514.49.2.395

Kim, P.H. and Aldrich, H.E. (2006), "Teams that work together, stay together: Resiliency of entrepreneurial teams.", Babson Kauffman Entrepreneurship Research Conference (BKERC), Babson College. 2002-2006.
Kleinbaum, A.M., Stuart, T.E. and Tushman, M.L. (2008), July). Communication (and coordination?) in a modern, complex organization (HBS Working Paper No. 09-004). Cambridge, MA: Organizational Behavior, Entrepreneurial Management, Harvard Business School.

Kraut, R.E., Fussell, S.R., Brennan, S.E. and Siegel, J. (2002), "Understanding effects of proximity on collaboration: Implications for technologies to support remote collaborative work". In: Hinds P, Kiesler S, eds, Distributed work. Cambridge: MIT Press. pp. 137-162.

Leenders, R.T.A.J. (1996), "Evolution of friendship and best friendship choices", The Journal of Mathematical Sociology, Vol. 21 No. 1-2, pp. 133-148. 10.1080/0022250X.1996.9990177

Lewis, K. (2000), "Is performance all in their mind(s)? The impact of transactive memory on knowledge worker team performance", Paper presented at the Academy of Management, Toronto, Canada.

Lin, N. (1982), "Social resources and instrumental action”. In: Marsden P. V, Lin N, eds, Social Structure and Network Analysis. Beverly Hills, CA: Sage. pp. 131-145.

Lincoln, J.R. and Miller, J. (1979), "Work and Friendship ties in organizations: a comparative analysis of relation networks", Administrative Science Quarterly, Vol. 24 No. 2, pp. 181-199. 10.2307/2392493

Love, P., Gunasekaran, A. and Li, H. (1998), "Concurrent engineering: a strategy for procuring construction projects", International Journal of Project Management, Vol. 16 No. 6, pp. 375-383.

Marsden, P.V. (1987), "Core discussion networks of Americans", American Sociological Review, Vol. 52 No. 1, pp. 122-113. 10.2307/2095397

McPherson, J.M. and Smith-Lovin, L. (1982), “Women and weak ties: differences by sex in the size of voluntary organizations", American Journal of Sociology, Vol. 87 No. 4, pp. 883-904. 10.1086/227525

McPherson, M., Smith-Lovin, L. and Cook, J.M. (2001), "Birds of a feather: Homophily in social networks", Annual Review of Sociology, Vol. 27 No. 1, pp. 415-444. 10.1146/annurev.soc.27.1.415

Meier, J. and Crocker, M. (2010), "Generation Y in the workforce: managerial challenges", The Journal of Human Resource and Adult Learning, Vol. 6 No. 1, p. 68.

Mollica, K.A., Gray, B. and Treviño, L.K. (2003), "Racial Homophily and Its Persistence in Newcomers' Social Networks", Organization Science, Vol. 14 No. 2, pp. 123-136. 10.1287/orsc.14.2.123.14994

Moore, D.R. and Dainty, A.R.J. (2001), "Intrateam boundaries as inhibitors of performance 
improvement in UK design and build projects: a call for change", Construction Management and Economics, Vol. 19 No. 6, pp. 559-562. 10.1080/01446190110055508

Paulus, P.B. and Nijstad, B.A. (2003), Group creativity: Innovation through collaboration. Oxford: Oxford University Press.

Pelled, L.H., Eisenhardt, K.M. Xin, K.R. and Janis, I.L. (1999), "Exploring the black box: an analysis of work group diversity, conflict, and performance", Administrative Science Quarterly No., Vol. 44 No. 1, pp. 1-28. 10.2307/2667029

Pruitt, D.G. and Drews, J.L. (1969), "The effect of time pressure, time elapsed, and the opponent's concession rate on behavior in negotiation", Journal of Experimental Social Psychology, Vol. 5 No. 1, pp. 43-60. 10.1016/0022-1031(69)90005-5

Pruitt, D.G. and Johnson, D.F. (1970), "Mediation as an aid to face saving in negotiation", Journal of Personality and Social Psychology, Vol. 14 No. 3, pp. 239-246. 10.1037/h0028883

Sampson, R.J. (1984), “Group Size, Heterogeneity, and Intergroup Conflict: A Test of Blau's Inequality and Heterogeneity", Social Forces, Vol. 62 No. 3, pp. 618-639. 10.2307/2578703

Schutz, W.C. (1955), "What makes groups productive?" Human Relations, Vol. 8 No. 4, pp. 429-465. 10.1177/001872675500800404

Shrum, W., Cheek, N.H. and Hunter, S.M. (1988), "Friendship in school: gender and racial homophily", Sociology of Education, Vol. 61 No. 4, pp. 227-239. 10.2307/2112441

South, S.J. (1988), "Sex Ratios, Economic Power, and Women's Roles: A Theoretical Extension and Em- pirical Test", Journal of Marriage and the Family, Vol. 50 No. 1, pp. 19-31. 10.2307/352424

South, S.J., Bonjean, C.M., Markham, W.T. and Corder, J. (1982), "Social structure and intergroup interaction: men and women of the federal bureaucracy", American Sociological Review, Vol. 47 No. 5, pp. 587-599. 10.2307/2095160

South, S.J., Bonjean, C.M., Markham, W.T. and Corder, J. (1983), "Female labor force participation and the organizational experiences of men and women", Sociology Quarter, Vol. 24, pp. 367-380.

Turner, J.C. (1987), Rediscovering the Social Group: A Self-Categorization Theory. Basil Blackwell: Oxford.

van der Kleij, R., Lijkwan, J.T.E., Rasker, P.C. and De Dreu, C.K.W. (2009), "Effects of time pressure and communication environment on team processes and outcomes in dyadic planning", International Journal of Human-Computer Studies, Vol. 67 No. 2009, pp. 411-423. 10.1016/j. ijhcs.2008.11.005

Verbrugge, L.M. (1977), "The structure of adult Friendship choices", Social Forces, Vol. 56 No. 2, pp. 576-597. 10.2307/2577741

Wagner, K.L. (2007), "Filling the gap: the generation gap in the workplace creates challenges for managers wanting to build a team", Journal of Property Management, Vol. 72 No. 5, pp. 29-35.

Yuan, Y.C. and Gay, G. (2006), "Homophily of network ties and bonding and bridging social capital in computer-mediated distributed teams", Journal of Computer-Mediated Communication, Vol. 11 No. 4 1062-1084. 10.1111/j.1083-6101.2006.00308.x 